\title{
Temperature dependence of spin Hall magnetoresistance in thin YIG/Pt films
}

\author{
S. R. Marmion, ${ }^{1,}{ }^{*}$ M. Ali, ${ }^{1}$ M. McLaren, ${ }^{2}$ D. A. Williams,${ }^{3}$ and B. J. Hickey ${ }^{1, \dagger}$ \\ ${ }^{1}$ School of Physics and Astronomy, University of Leeds, Leeds LS2 9JT, United Kingdom \\ ${ }^{2}$ Institute of Materials Research, SPEME, University of Leeds, Leeds LS2 9JT, United Kingdom \\ ${ }^{3}$ Hitachi Research Laboratory, Cambridge CB3 OHE, United Kingdom
}

(Received 12 February 2014; revised manuscript received 30 April 2014; published 13 June 2014)

\begin{abstract}
We report on the temperature dependence of the recently discovered spin Hall magnetoresistance in a yttrium iron garnet (YIG)/platinum (Pt) thin film. The YIG/Pt layers are an ideal choice as the combination of an insulating magnetic material and the high spin-orbit interaction in Pt gives a relatively large magnetoresistance and no electrical conduction occurs in the YIG. The temperature dependence of the magnetoresistance was measured between $1.4 \mathrm{~K}$ and $280 \mathrm{~K}$ from which the temperature dependence of the spin diffusion length in $\mathrm{Pt}$ has been extracted. We found that the best agreement between our data and the recently published [Chen et al., Phys. Rev. B 87, 144411 (2013)] theory of the spin Hall magnetoresistance is given by an assumed Elliot-Yafet mechanism of spin relaxation with temperature-independent spin Hall angle and spin mixing conductance. The best estimate for the spin diffusion length returns values between 0.57 and $3.85 \mathrm{~nm}$.
\end{abstract}

DOI: 10.1103/PhysRevB.89.220404

PACS number(s): 75.70.-i, 72.15.Gd, 75.76.+j, 71.70.Ej

\section{INTRODUCTION}

The recently discovered spin Hall magnetoresistance (SMR) [1] occurs in nonmagnetic metals with high spin-orbit interaction such as platinum, when grown on a magnetic material. This was first observed in a yttrium iron garnet (YIG)/platinum $(\mathrm{Pt})$ thin film where the YIG, being a magnetic insulator, is an ideal candidate as there will be no problem with current shunting. The SMR effect is due to the simultaneous action of both the spin Hall (SHE) [2] and inverse spin Hall (ISHE) [3-5] effects. The spin-orbit interaction of the platinum acts on the applied charge current giving rise to the SHE generating a spin current transverse to the direction of the charge current. This spin current causes spin accumulation at the YIG/Pt interfaces. Spins that reflect from this interface are then deflected by the ISHE generating a small additional charge current that makes a contribution to the total resistance of the electrode which depends on the applied magnetic field. The magnetoresistance arises because the magnetization of the YIG at one of the platinum interfaces can either enhance the reflection of the spins or absorb angular momentum through spin transfer torque depending on the relative orientation of the magnetization $\mathbf{M}$ and the electron spin $\mathbf{s}$. In the latter case this leads to a reduction in the spins available to undergo ISHE and so reduces the contribution of the spin current to the charge current.

As there are many other Hall and magnetoresistive effects, it is important to confirm the origin of any observed change in resistance and the details of how applied magnetic fields affect them. Recently the possibility of a magnetic proximity effect, whereby a magnetic moment is induced in a metal overlayer, has been investigated in YIG/Pt bilayers. It might be expected that since YIG is an insulator it is unlikely that it will be able to induce a magnetic moment in a metal since there is no possibility for spin-polarized charge carriers to pass between YIG and the Pt. Geprägs et al. [6] found using

*pysrm@leeds.ac.uk

†b.j.hickey@leeds.ac.uk
$\mathrm{X}$-ray magnetic circular dichroism (XMCD) that if there were a moment induced in $\mathrm{Pt} / \mathrm{YIG}$, it was 30 times smaller than the induced moment found in a reference $\mathrm{Pt} / \mathrm{Fe}$ sample. On the other hand, Lu et al. [7] suggest that there is an induced moment for a sample of Pt/YIG. Thus it might be possible that a thin layer of Pt may exhibit an anisotropic magnetoresistance (AMR) so it is important to demonstrate that the SMR is measured and not the AMR [8]. It is also prudent be take account of other possible effects such as the Nernst effect [9-11].

So far experiments observing this SMR in YIG/Pt films have made the YIG by liquid phase epitaxy [1] or pulsed laser deposition [8]; however we have used radio frequency (RF) magnetron sputtering, a well established and relatively cheap method that would be the ideal choice for use in any possible industrial applications [12].

Our aim in this Rapid Communication is to measure the temperature dependence of the SMR with a view to determining the mechanism of spin relaxation in the Pt and we suggest that a single spin relaxation mechanism $[11,13,14]$ best describes the temperature dependence of the SMR.

\section{EXPERIMENTAL DETAILS}

YIG was grown using RF sputtering in an argon/oxygen atmosphere on substrates of (111) gadolinium gallium garnet (GGG) to a thickness of $45 \pm 2 \mathrm{~nm}$ at a pressure of 2.4 mTorr. This substrate was chosen as it has the same crystal structure and similar lattice constant to YIG $(12.383 / 12.376 \AA$ GGG/YIG) and the thermal expansion coefficients are similar. As deposited the YIG was nonmagnetic and so was annealed ex situ at $850^{\circ}$ for 2 hours. The sample was heated at a rate of $7^{\circ}$ per minute to avoid any unnecessary strain on the film and similarly, slowly cooled. Platinum was deposited by DC magnetron sputtering at a growth rate of $0.20 \pm 0.01 \AA / \mathrm{s}$. A shadow mask was used to create the two thin electrodes $3 \mathrm{~mm}$ long, $100 \mu \mathrm{m}$ wide, and $2.5 \mathrm{~nm}$ thick each with contact pads at the end as shown in Fig. 1. Both YIG and Pt thicknesses were measured by $\mathrm{x}$-ray reflectivity. The resistivity of the platinum was $56 \pm 5 \mu \Omega \mathrm{cm}$, well above the bulk value of $10.6 \mu \Omega \mathrm{cm}$. 


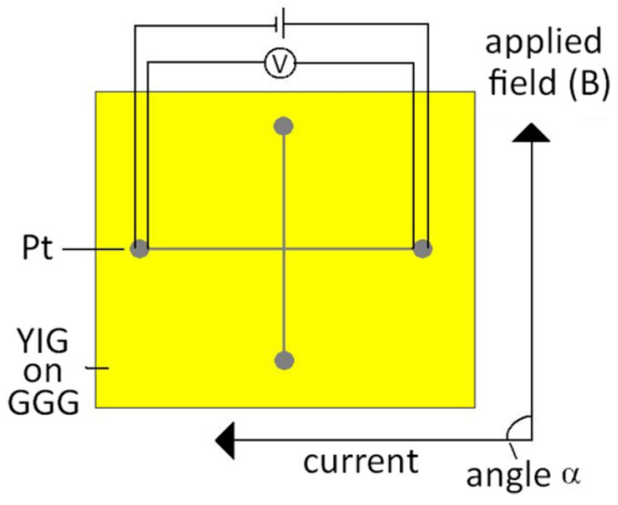

FIG. 1. (Color online) Illustration of the YIG sample and the Pt wires for a 4-probe method on an $8 \times 8 \mathrm{~mm}$ square substrate.

For the SMR effect to be produced it is necessary to have a good quality interface between the YIG and the platinum to allow spin transfer across the interface [15]. A series of YIG/Pt films were prepared into cross sections for observation in a transmission electron microscope (TEM). The films were stacked with silicon wafers and held together with epoxy. A circular core of the stack was extracted and encapsulated in a brass ring before being cut into cross sections with a diamond saw. These cross sections were polished, dimpled, and finally ion polished to create electron transparent regions 20-50 nm thick. The characterization was performed on a FEI Tecnai TF20 operated at $200 \mathrm{kV}$ using high-resolution TEM (HRTEM) and scanning TEM (STEM) electron dispersive $\mathrm{X}$-ray spectroscopy (EDX). The bright-field images show that the crystal structure of the YIG is as expected and that this structure is maintained right up to the interface with the platinum. High-quality crystals have been produced and Fig. 2

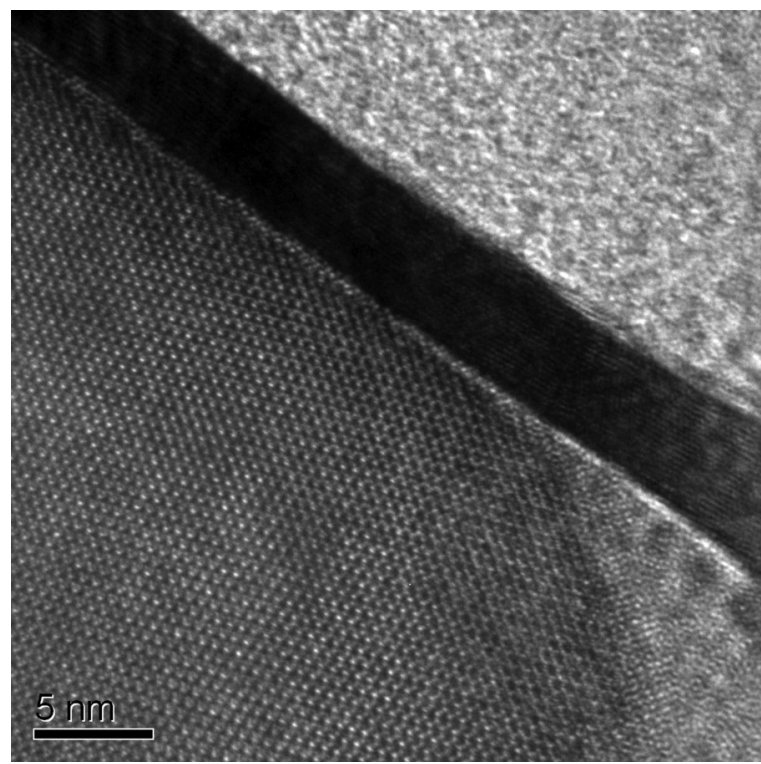

FIG. 2. TEM image of a YIG/Pt interface. Here the platinum is the black strip and the YIG underneath this. The breakdown in crystal structure to the lower right corner is due to the ion beam during sample preparation, consistent with other observations of ion polishing damaging the structure.

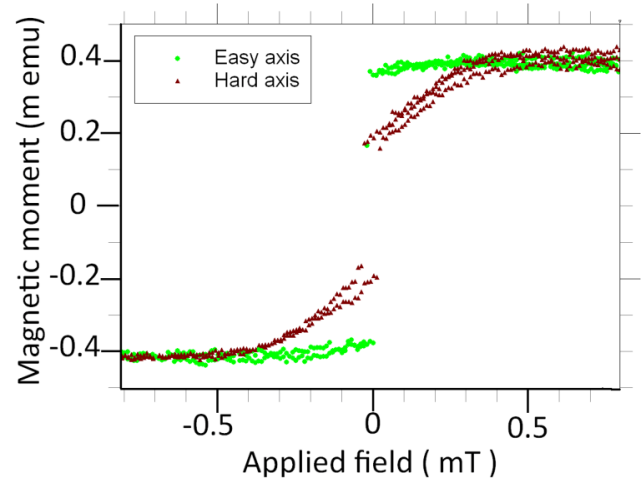

FIG. 3. (Color online) VSM data for the two different orientations measured at $250 \mathrm{~K}$ showing the magnetic easy axis lies along the line of one of the electrodes and the hard axis along the other.

demonstrates a typical YIG/Pt interface and the YIG can be seen as a single crystal. STEM-EDX mapping using the $\mathrm{O}-\mathrm{K} \alpha$ peak found no noticeable change in the oxygen content at the YIG/GGG interface, suggesting very little or no oxygen deficiency in the YIG layer.

To measure the temperature dependence the sample was wire bonded to the contact pads to create a 4-point probe measurement using a constant DC current of $0.1 \mathrm{~mA}$.

\section{RESULTS}

Vibrating sample magnetometer (VSM) measurements show (Fig. 3) there is an anisotropy in the YIG film believed to have been induced during growth by the in situ permanent magnets used for that purpose. The magnetization of the YIG was found to be $140 \pm 10$ e.m.u/cc as expected for bulk material. In the hard axis the magnetic reversal is characterized by domain wall motion followed by a slow rotation of the magnetization whereas along the easy axis the main reversal mechanism is domain wall motion. The coercivity is low with a value of $25 \pm 2 \mu \mathrm{T}$.

Figure 4 shows a typical magnetoresistance along the YIG hard axis $\left(\alpha=90^{\circ}\right)$ where it can be seen that the SMR saturates at an applied field of $0.5 \mathrm{mT}$ which coincides with the saturation field of the hard axis of the YIG in Fig. 3.

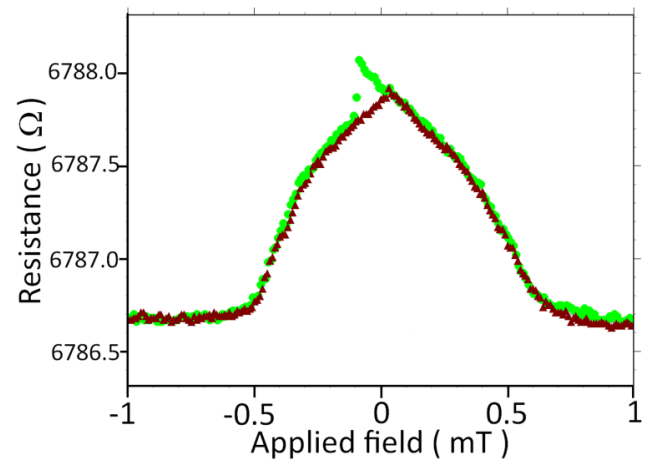

FIG. 4. (Color online) Example of a field sweep at $250 \mathrm{~K}$ with $\alpha=90^{\circ}$. The sweep starts at the maximum positive applied field following the green curve and retraced back with the brown curve. 


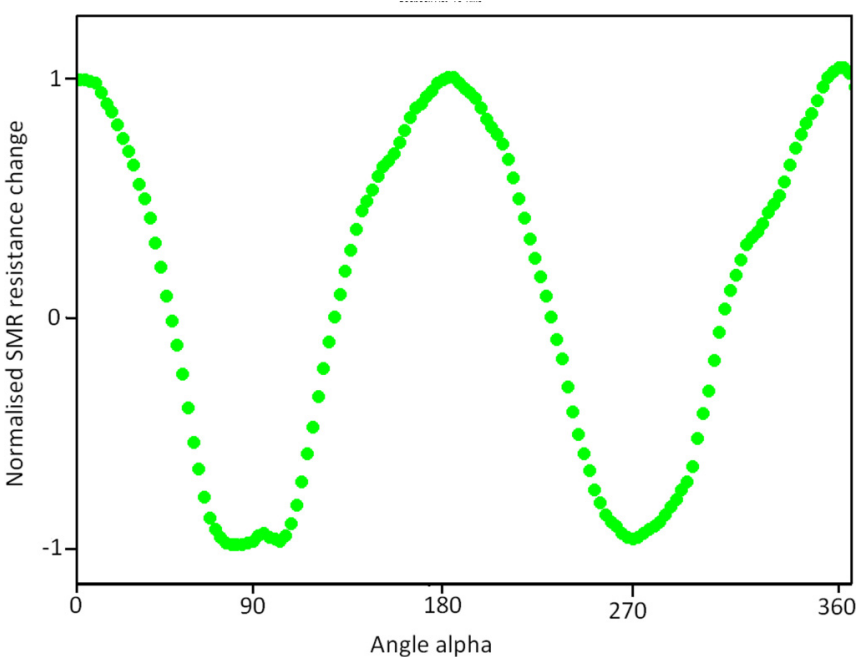

FIG. 5. (Color online) Normalized SMR resistance change as a function of angle $\alpha$ as the sample is rotated in a saturating magnetic field in the plane of the film.

It has been suggested [11] that if the Pt is polarized due to the proximity to YIG, it may exhibit the AMR. The angular dependence of the SMR was measured in a saturating magnetic field in the plane of the YIG film as the sample was rotated through $360^{\circ}$ as shown in Fig. 5. The SMR depends on the angle between $\mathbf{M}$ and the spin accumulation at the YIG/Pt interface whereas the AMR depends on the angle between $\mathbf{M}$ and $\mathbf{j}$. The AMR is larger when $\mathbf{M}$ and $\mathbf{j}$ are parallel and therefore, if this measurement is the AMR we would expect that the angular dependence should be offset by $90^{\circ}$ from what we observe. The samples were then subjected to a perpendicular magnetic field where the magnetization was rotated out of the plane of the film in two conditions: (a) where the angle between $\mathbf{M}$ and $\mathbf{j}$ is constant and equal to $90^{\circ}$ and (b) where $\mathbf{M}$ is rotated out of plane from initially in-plane and parallel to $\mathbf{j}$. Condition (a) will produce no AMR since $\mathbf{M}$ and $\mathbf{j}$ are always perpendicular. However, it will produce an SMR at $90^{\circ}$ intervals as $\mathbf{M}$ and the spin quantization axis go in and out of alignment and this is what is observed. On the other hand, condition (b) should produce no SMR but will produce the maximum AMR as $\mathbf{M}$ and $\mathbf{j}$ change from parallel to perpendicular. In this case we measured no change in MR which is convincing evidence that the effect is SMR as shown in Fig. 6. Here the blue curve represents condition (a) and the green curve condition (b). These data are consistent with other published results $[1,16]$ on similar samples.

From the current theory of the SMR Eq. (1) [16] gives the maximum change in resistivity $\rho$ for the condition of M rotating from parallel to the spin quantization axis to perpendicular. This change in resistivity is related to the spin diffusion length $\lambda$, the spin Hall angle $\theta_{s h}$, and the spin mixing conductance $G ; \sigma$ is the platinum conductivity and $d$ is the $\mathrm{Pt}$ thickness:

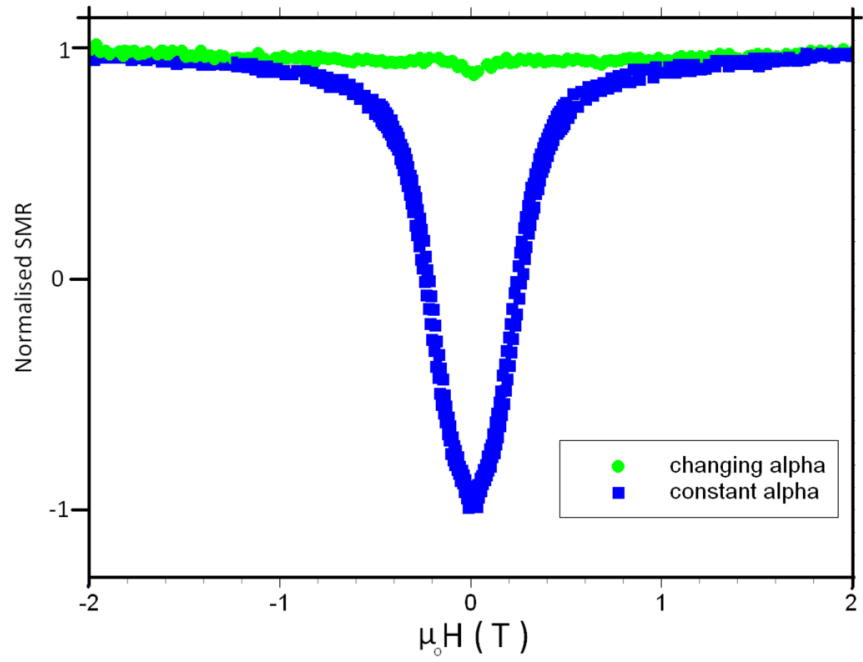

FIG. 6. (Color online) These data demonstrate that the measured effect is the SMR and not AMR. The squares are data where the angle between $\mathbf{M}$ and $\mathbf{j}$ is constant and equal to $90^{\circ}$ rotating from in plane and transverse to $\mathbf{j}$ to out of plane; therefore this is the SMR. The AMR is a constant if the angle between $\mathbf{M}$ and $\mathbf{j}$ is unchanged. The circles are data where the angle between $\mathbf{M}$ and $\mathbf{j}$ rotates from in plane and parallel to $\mathbf{j}$ to out of plane. In this case the AMR should vary to its maximum extent and there should be no SMR. These two curves indicate that there is only SMR and no AMR measured in this sample.

Our main objective is to understand the temperature dependence of the SMR. There are three quantities that are potentially temperature dependent in this equation: $\lambda, \theta_{s h}$, and $G$. The spin mixing conductance $G$ is a complex quantity determined by the reflection and transmission coefficients of the YIG/Pt interface. In fact, $G$ is dominated by the the real part of the reflection coefficient in nearly all samples [16] relevant to this discussion, is independent of thickness, and has recently been shown [17] to take the value $1 \times 10^{15} \Omega^{-1} \mathrm{~m}^{-2}$. Since $G$ depends on the reflection of spins from the YIG/Pt interface and we are far from the critical temperature of YIG (the bulk critical temperature is $550 \mathrm{~K}$ ) in our measurements, we anticipate that $G$ will not be temperature dependent. The spin Hall angle is given by $\theta_{s h}=\sigma_{\text {spin }} / \sigma$ and where $\sigma_{\text {spin }}$ is the spin conductivity and is related to the spin conductance in the usual way. The spin conductance is temperature dependent [18] and so is the ordinary conductivity of Pt. Thus $\theta_{s h}$ could be temperature dependent but could also be independent of temperature if the dependencies of the ratio are compensating.

The spin diffusion length by contrast has been shown to have a clear temperature dependence [14] that depends on the mechanism of spin relaxation. The mechanism we expect to account for spin relaxation in Pt is the Elliot-Yafet spin-orbit scattering model $[19,20]$. The spin diffusion length is defined as the product of the diffusion constant $D$ and the spin-flip relaxation time $\tau_{s f}$ :

$$
\frac{\Delta \rho}{\rho}=\theta_{s h}^{2} \frac{\lambda}{d} \frac{2 \lambda G \tanh ^{2} \frac{d}{2 \lambda}}{\sigma+2 \lambda G \operatorname{coth} \frac{d}{\lambda}} .
$$

$$
\lambda=\sqrt{D \tau_{s f}} .
$$




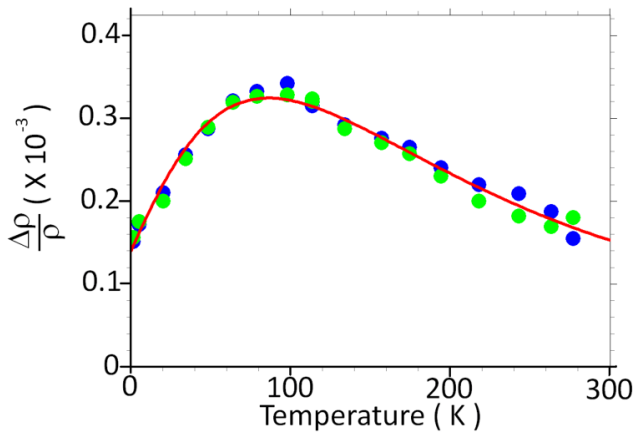

FIG. 7. (Color online) Temperature dependence of the SMR. The points are measured data showing forward (green) and reverse (blue) magnetic field sweeps between 1.4 and $280 \mathrm{~K}$. The solid line is a fit to the data using Eq. (1) and the Elliot-Yafet assumption that the spin diffusion length is proportional to $1 / T$.

The diffusion constant follows the reciprocal temperature dependence of the resistivity:

$$
D(T) \propto \frac{1}{\rho(T)} .
$$

Within the Elliot-Yafet model, $\tau_{s f}$ has the same temperature dependence as the momentum relaxation time $\tau_{p}$ and this also follows the reciprocal of temperature dependence of the resistivity:

$$
\tau_{s f}(T) \propto \tau_{p}(T) \propto \frac{1}{\rho(T)} .
$$

So we can assume that the spin diffusion length is proportional to the conductivity directly or we can also say that the temperature dependence of the spin diffusion length will go as the temperature dependence of phonon scattering (the dominant scattering mechanism for momentum relaxation); hence,

$$
\lambda \propto \frac{1}{T} .
$$

In our analysis, we shall explore both of these possibilities as well as the temperature dependence of the spin Hall angle.

Figure 7 shows the forward and reverse field sweeps at different temperatures and the solid line (red) is the best fit to the data using Eq. (1). The best fit was obtained with a spin Hall angle (assumed to be independent of temperature) of $0.044 \pm 0.002$ and a $G$ of $7.57 \pm 0.01 \times 10^{14} \Omega^{-1} \mathrm{~m}^{-2}-$ both of which are reasonable $[8,17,20]$. Figure 8 shows the temperature-dependent $\lambda$ where we can see an obvious fit that matches our assumption of the $1 / T$ dependence highlighted by the solid (red) line.

In the process of determining the best fit, we explored the possibilities for the other parameters. We considered the case that the spin diffusion length is proportional to the electrical conductivity and took the spin conductivity to be constant, i.e., that the spin Hall angle has a temperature dependence determined by that of the conductivity. In this case we could not fit the data at all and unreasonable spin diffusion lengths of between $0.13-0.16 \mathrm{~nm}$ were returned that were temperature independent. In fact it was impossible to fit the data in any variation of the parameters while keeping the condition that $\lambda \propto \sigma$. Perhaps this is to be expected. Our Pt is only $2.5 \mathrm{~nm}$ thick where the resistivity is probably dominated by surface

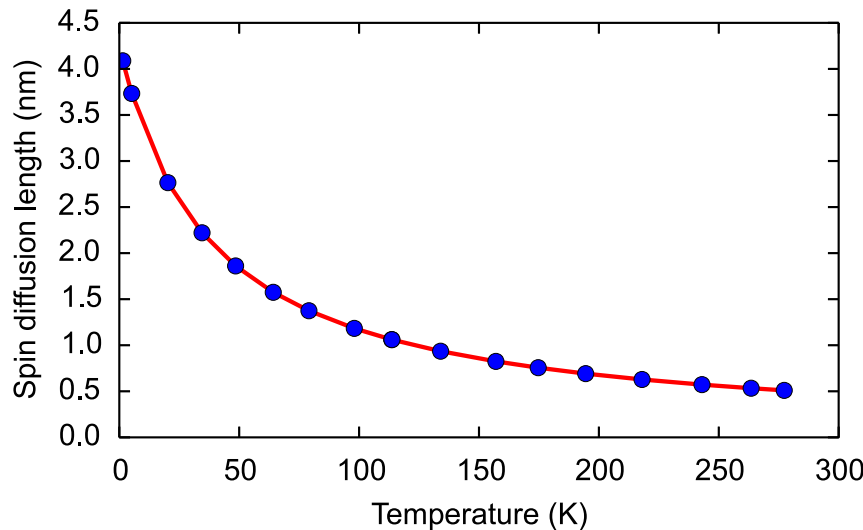

FIG. 8. (Color online) The spin diffusion length derived from fit and based on the Elliot-Yafet mechanism.

scattering whereas the relaxation mechanism for Elliot-Yafet is a bulk effect that relies on the spin-orbit interaction.

We considered a temperature dependent spin Hall angle by assuming $\sigma_{\text {spin }}$ to be constant and keeping $\lambda \propto 1 / T$. A reasonable fit is obtained with $\theta_{s h}$ varying linearly from 0.046 to 0.054 between low temperatures and room temperature, respectively, and the constant value of $9.27 \pm 0.03 \times 10^{4} \Omega^{-1} \mathrm{~m}^{-1}$ for $\sigma_{\text {spin }}$. However, allowing the spin conductivity to change with temperature compensates the change in electrical conductivity and thus keeps the spin Hall angle constant improving this fit still further. The best fit returns a linear temperature dependence of $\sigma_{\text {spin }}$ varying from $7.61 \pm 0.03 \times 10^{4} \Omega^{-1} \mathrm{~m}^{-1}$ at room temperature to $8.83 \pm 0.03 \times 10^{4} \Omega^{-1} \mathrm{~m}^{-1}$ at low temperatures-a $17 \%$ increase over the temperature range. We expect $\sigma_{\text {spin }}$ to vary in a similar way to $\sigma_{\text {she }}$, the spin Hall conductivity, and have therefore compared our results to those measured by Vila et al. [18] where they found that $\sigma_{\text {she }}$ increased linearly by $30 \%$ over a similar temperature range which agrees well with our results.

The limits of Eq. (1) need to be considered with respect to $G$ to see what the effect of changing this value has on the fit in Fig. 7 and also on the spin diffusion length. By taking the limits as $G \gg(\ll) \frac{\sigma}{2 \lambda}$ and then fitting the same $1 / T$ dependence to the now adapted version of Eq. (1), the fits in Fig. 9 are found. From the best fits $\frac{\sigma}{2 \lambda}$ is linear in temperature and ranges from 0.2 to $1.6 \times 10^{15}$ from low to high temperature suggesting that

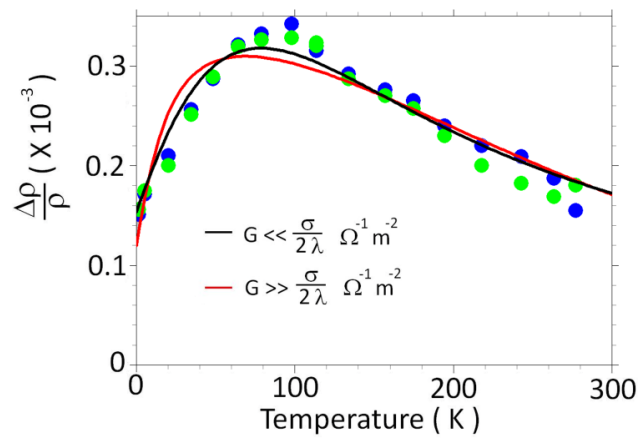

FIG. 9. (Color online) Alternative fits for the SMR where the spin mixing conductance is taken to extremes. 
a $G$ of order $10^{15}$ is the right value and Fig. 9 is clearly a poorer fit compared to Fig. 7.

Finally we comment on the size of the spin diffusion length at room temperature. Our best fit returns a value of about $0.5 \mathrm{~nm}$ which is shorter than expected by a factor of about 3 ; however the resistivity of our very thin Pt is about $56 \mu \Omega \mathrm{cm}$ which suggests a mean-free path that is similar in magnitude to the spin diffusion length. This is clearly on the limits of where we would expect the Elliot-Yafet model to apply. The literature reports of the spin diffusion length of $\mathrm{Pt}$ are quite variable $(1.5-14 \mathrm{~nm})$ and there is considerable discussion about how best to determine it $[20,21]$. We have explored the flexibility of the parameters and cannot find sensible values that will fit the data with a larger spin diffusion length at room temperature. Possible explanations for this might be that near the interface the $\mathrm{Pt}$ is polarized and we have not taken this into account or that the SMR is a purely interface effect and the bulklike spin-orbit scattering of the Elliot-Yafet theory does not apply.

\section{CONCLUSIONS}

The temperature dependence of the SMR has been measured in a YIG/Pt system and we have shown that the data can be explained by the existing theory for the effect and a temperature-dependent spin diffusion length that is consistent with the Elliot-Yafet mechanism for spin-flip relaxation. This fit indicates that the spin diffusion length of the platinum range is $4-0.5 \mathrm{~nm}$ over the $0-280 \mathrm{~K}$ region assuming reasonable values for the spin Hall angle $(0.44)$ and the spin mixing conductance $7.57 \pm 0.01 \times 10^{14} \Omega^{-1} \mathrm{~m}^{-2}$.

\section{ACKNOWLEDGMENTS}

We would like to thank G. E. W. Bauer, C. Back, and R. J. Hicken for valuable discussions. This work was funded by the Engineering and Physics Sciences Research Council of the UK and Hitachi Cambridge Laboratory.
[1] H. Nakayama, M. Althammer, Y.-T. Chen, K. Uchida, Y. Kajiwara, D. Kikuchi, T. Ohtani, S. Geprägs, M. Opel, S. Takahashi, R. Gross, G. E. W. Bauer, S. T. B. Goennenwein, and E. Saitoh, Phys. Rev. Lett. 110, 206601 (2013).

[2] J. E. Hirsch, Phys. Rev. Lett. 83, 1834 (1999).

[3] T. Kimura, Y. Otani, T. Sato, S. Takahashi, and S. Maekawa, Phys. Rev. Lett. 98, 156601 (2007).

[4] C. Hahn, G. de Loubens, O. Klein, M. Viret, V. V. Naletov, and J. Ben Youssef, Phys. Rev. B 87, 174417 (2013).

[5] E. Saitoh, M. Ueda, H. Miyajima, and G. Tatara, Appl. Phys. Lett. 88, 182509 (2006).

[6] S. Geprägs, S. Meyer, S. Altmannshofer, M. Opel, F. Wilhelm, A. Rogalev, R. Gross, and S. T. B. Goennenwein, Appl. Phys. Lett. 101, 262407 (2012).

[7] Y. M. Lu, Y. Choi, C. M. Ortega, X. M. Cheng, J. W. Cai, S. Y. Huang, L. Sun, and C. L. Chien, Phys. Rev. Lett. 110, 147207 (2013).

[8] M. Althammer, S. Meyer, H. Nakayama, M. Schreier, S. Altmannshofer, M. Weiler, H. Huebl, S. Geprägs, M. Opel, R. Gross, D. Meier, C. Klewe, T. Kuschel, J.-M. Schmalhorst, G. Reiss, L. Shen, A. Gupta, Y.-T. Chen, G. E. W. Bauer, E. Saitoh, and S. T. B. Goennenwein, Phys. Rev. B 87, 224401 (2013).

[9] M. Weiler, M. Althammer, F. D. Czeschka, H. Huebl, M. S. Wagner, M. Opel, I.-M. Imort, G. Reiss, A. Thomas, R. Gross, and S. T. B. Goennenwein, Phys. Rev. Lett. 108, 106602 (2012).
[10] J. J. Hauser, Phys. Rev. 187, 580 (1969).

[11] S. Y. Huang, X. Fan, D. Qu, Y. P. Chen, W. G. Wang, J. Wu, T. Y. Chen, J. Q. Xiao, and C. L. Chien, Phys. Rev. Lett. 109, 107204 (2012).

[12] Y.-M. Kang, S.-H. Wee, S.-I. Baik, S.-G. Min, S.-C. Yu, S.-H. Moon, Y.-W. Kim, and S.-I. Yoo, J. Appl. Phys. 97, 10A319 (2005).

[13] Y. Ando, K. Kasahara, S. Yamada, Y. Maeda, K. Masaki, Y. Hoshi, K. Sawano, M. Miyao, and K. Hamaya, Phys. Rev. B 85, 035320 (2012).

[14] T. Kimura, T. Sato, and Y. Otani, Phys. Rev. Lett. 100, 066602 (2008).

[15] B. Heinrich, C. Burrowes, E. Montoya, B. Kardasz, E. Girt, Y.-Y Song, Y. Sun, and M. Wu, Phys. Rev. Lett. 107, 066604 (2011).

[16] Y.-T. Chen, S. Takahashi, H. Nakayama, M. Althammer, S. T. B. Goennenwein, E. Saitoh, and G. E. W. Bauer, Phys. Rev. B 87, 144411 (2013).

[17] M. Weiler, M. Althammer, M. Schreier, J. Lotze, M. Pernpeintner, S. Meyer, H. Huebl, R. Gross, A. Kamra, J. Xiao, Y.-T. Chen, H. J. Jiao, G. E. W. Bauer, and S. T. B. Goennenwein, Phys. Rev. Lett. 111, 176601 (2013).

[18] L. Vila, T. Kimura, and Y. C. Otani, Phys. Rev. Lett. 99, 226604 (2007).

[19] J. Fabian and S. Das Sarma, Phys. Rev. Lett. 81, 5624 (1998).

[20] L. Liu, R. A. Buhrman, and D. C. Ralph, arXiv:1111.3702.

[21] J. Bass and W. P. Pratt, Jr., J. Phys.: Condens. Matter 19, 183201 (2007). 